\title{
Constraining anomalous EeV ANITA detections with PeV neutrinos
}

\author{
Ibrahim Safa ${ }^{* 1}$, Alex Pizzuto ${ }^{1}$, Carlos A. Argüelles ${ }^{2}$, Francis Halzen ${ }^{1}$, Raamis \\ Hussain $^{1}$, Ali Kheirandish ${ }^{1}$, and Justin Vandenbroucke ${ }^{1}$ \\ ${ }^{1}$ Department of Physics and Wisconsin IceCube Particle Astrophysics Center, University of \\ Wisconsin, Madison, WI 53706, USA \\ ${ }^{2}$ Dept. of Physics, Massachusetts Institute of Technology, \\ Cambridge, MA 02139, USA \\ E-mail: isafa@wisc.edu
}

\begin{abstract}
The ANtarctic Impulsive Transinet Antenna (ANITA) recently reported two $\sim$ EeV showers which, at first glance, could be interpreted as emerging tau neutrinos. Their emergence angle was shown to be in tension with the expected angular distribution from isotropic astrophysical neutrinos. However, localized emission from a point source in the event's direction can evade diffuse bounds. In this work, we present an updated calculation of the tau-regeneration effect using a newly developed Monte Carlo package, TauRunner. We describe the algorithm and the softwares capabilities, and apply it to the anomalous ANITA detections. We show that any flux from the direction of the ANITA events should be accompanied by secondary tau-neutrinos detectable at IceCube. We derive the maximum allowed secondary flux at IceCube and compare it to the flux required to detect one event at ANITA. We find that these fluxes are in severe tension, requiring a significant over-fluctuation to account for the anomalous event in 2014.
\end{abstract}

36th International Cosmic Ray Conference -ICRC2019-

July 24th - August 1st, 2019

Madison, WI, U.S.A.

${ }^{*}$ Speaker. 


\section{Introduction}

Astrophysical neutrinos, both despite and because of their elusiveness, provide a direct probe to some of the most energetic processes in the Universe. We can see farther into some of the most violent of objects with neutrinos than we can with any other messenger. As such, features in the astrophysical neutrino spectrum encode invaluable information about cosmic-ray acceleration, mass composition, production sites, surrounding environments, and more. Since the completion of its construction in 2011, IceCube, a cubic-kilometer Cherenkov detector in the South Pole, has measured an astrophysical flux of neutrinos extending to $10 \mathrm{PeVs}$ [1]. At higher energies, experiments like ARA and ANITA have attempted to utilize the South Pole ice in other ways to detect radio emission from the highest energy neutrinos predicted, at scales approaching EeVs, and have set limits on cosmogenic neutrino fluxes [2,3]. On top of that, next-generation proposed experiments like GRAND and POEMMA go beyond the ice, using mountains and the Earth's atmosphere, respectively, as their effective volumes [4, 5]. There exists, however, a complementary channel that would take advantage of existing detectors like IceCube to probe the $\mathrm{EeV}$ universe. Particularly, tau-neutrinos traversing the earth produce a tau when they undergo a charged-current interaction, which subsequently decays emitting a daughter tau-neutrino. These secondary neutrinos emerge at energies between $100 \mathrm{TeV}$ and $10 \mathrm{PeV}$, a range where IceCube is quite sensitive. In this work we describe the prescription and associated uncertainties of lepton propagation through matter and introduce a monte carlo package, TauRunner, that propagates taus and tau-neutrinos through the earth. We show the energy distribution of secondary neutrinos after earth propagation for several injected fluxes and demonstrate the capability of Cherenkov detectors like IceCube to probe the $\mathrm{EeV}$ universe with $\mathrm{PeV}$ neutrinos. In particular, we use the regeneration effect [6] to constrain the anomalous ANITA events, and show that a tau-neutrino interpretation assuming localized emission is disfavored.

\section{Leptons through the Earth}

Although neutrino cross section measurements are available from $\mathrm{GeV}$ to $\mathrm{PeV}$ energies, [7, $8,9,10,11,12]$, they stop short of the region of interest for this work, and uncertainties from extrapolations disagree at the highest relevant energies. The disagreement is due to their respective treatment of the nucleon structure function. This function describes the fraction of the proton's momentum that a quark carries, and cannot be derived from first principles. Instead, we rely on empirical measurements of this function, which only extend down to Bjorken- $x=\mathscr{O}\left(10^{-6}\right)$, requiring extrapolations to lower values. Perturbative QCD calculations, although in good agreement with each other, exhibit a growth of the neutrino-nucleon cross section at high energies that violates the Froissart bound. A $\ln ^{2}(\mathrm{~s})$ extrapolation of low-energy measurements using a dipole model of the nucleon has been used in the past to address this issue. These approaches have been shown to be in good agreement with the total proton-proton cross-section measurements from Auger. In this work, we use [13] as a benchmark, which implements a dipole model presented here [14]. The cross section is shown in figure 1 . 


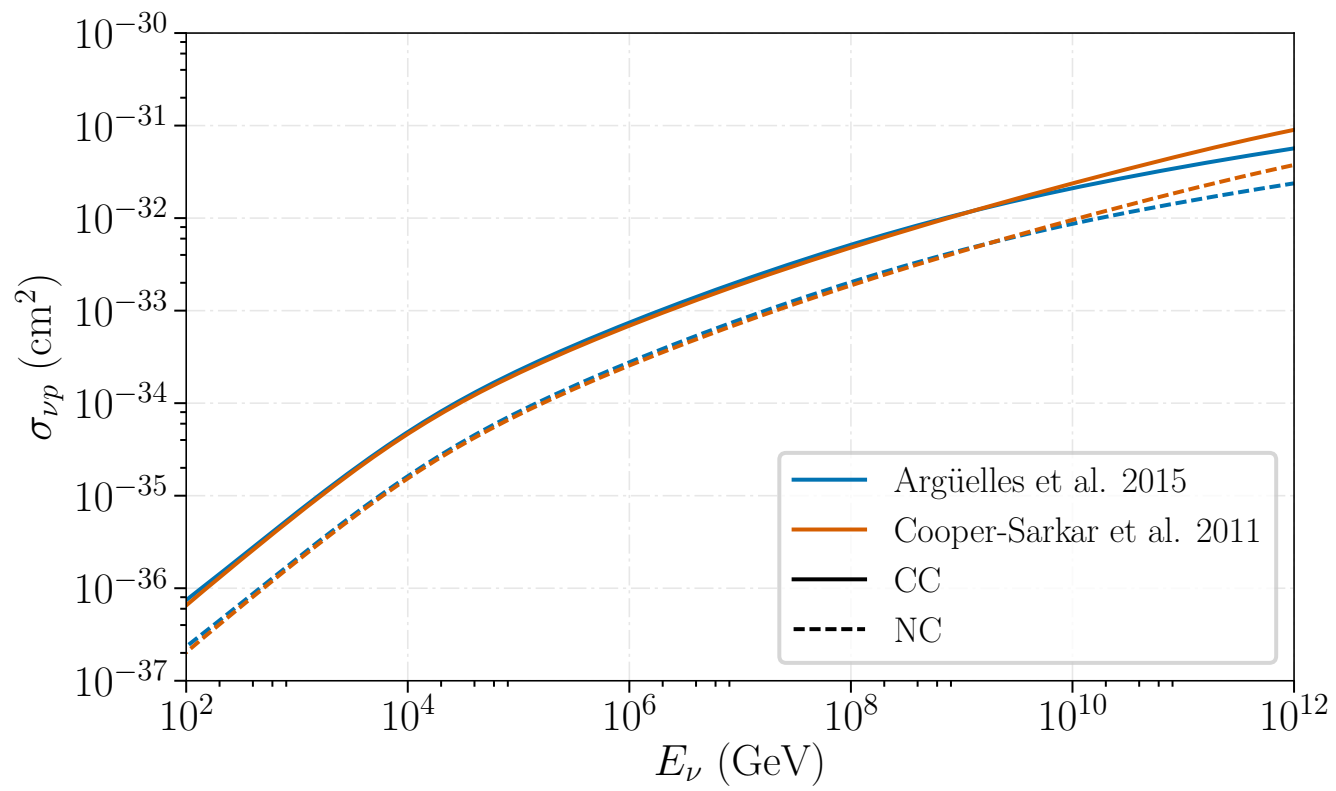

Figure 1: The neutrino-proton cross section as a function of energy. Solid (dashed) lines correspond to charged-current (neutral-current) cross sections. Blue lines [13] correspond to the model used for the results of this work. Orange lines [15] are implemented in the software as well and can be chosen by the user.

Compared to the muon mass, the tau mass is roughly seventeen times larger. Weak decays are proportional to $\mathrm{m}^{5}$, which means that the tau lifetime is many orders of magnitude smaller. For this reason, tau energy losses in most applications below $10 \mathrm{PeV}$ can be safely neglected, as they decay promptly. However, the mean interaction length is comparable to the decay length around 1 $\mathrm{EeV}$. Therefore, for this work, a careful treatment of tau losses is required. Taus and muons lose energy through the same processes, although taus at these energies are immediately in the radiative regime. This means that ionization, which scales like $\ln (E)$ is sub-dominant. Bremsstrahlung and pair-production are also sub-dominant to photo-nuclear interactions for taus, which dominate above an EeV [16], and grow with energy. Given that the photo-nuclear cross section depends on the nucleon structure function, the same uncertainties as in the neutrino cross section arise, and have to be addressed here. We use the same model of the nucleon structure function chosen for the neutrino-proton cross-section, and implement it in a publicly available charged-lepton propagation tool, Muon Monte Carlo (MMC) [17]. This modified version of MMC is then used to propagate taus, accounting for the stochastic nature of their losses.

\section{TauRunner}

To propagate high-energy neutrinos and taus through the Earth, both of which involve stochastic processes, we developed a python Monte Carlo package, TauRunner. This software takes in as input an initial neutrino energy and medium properties. The algorithm then calculates the neutrino's interaction length using the total cross-section and given medium properties. We use the Preliminary Reference Earth Model (PREM) [18] for the work presented here. Then, using the 


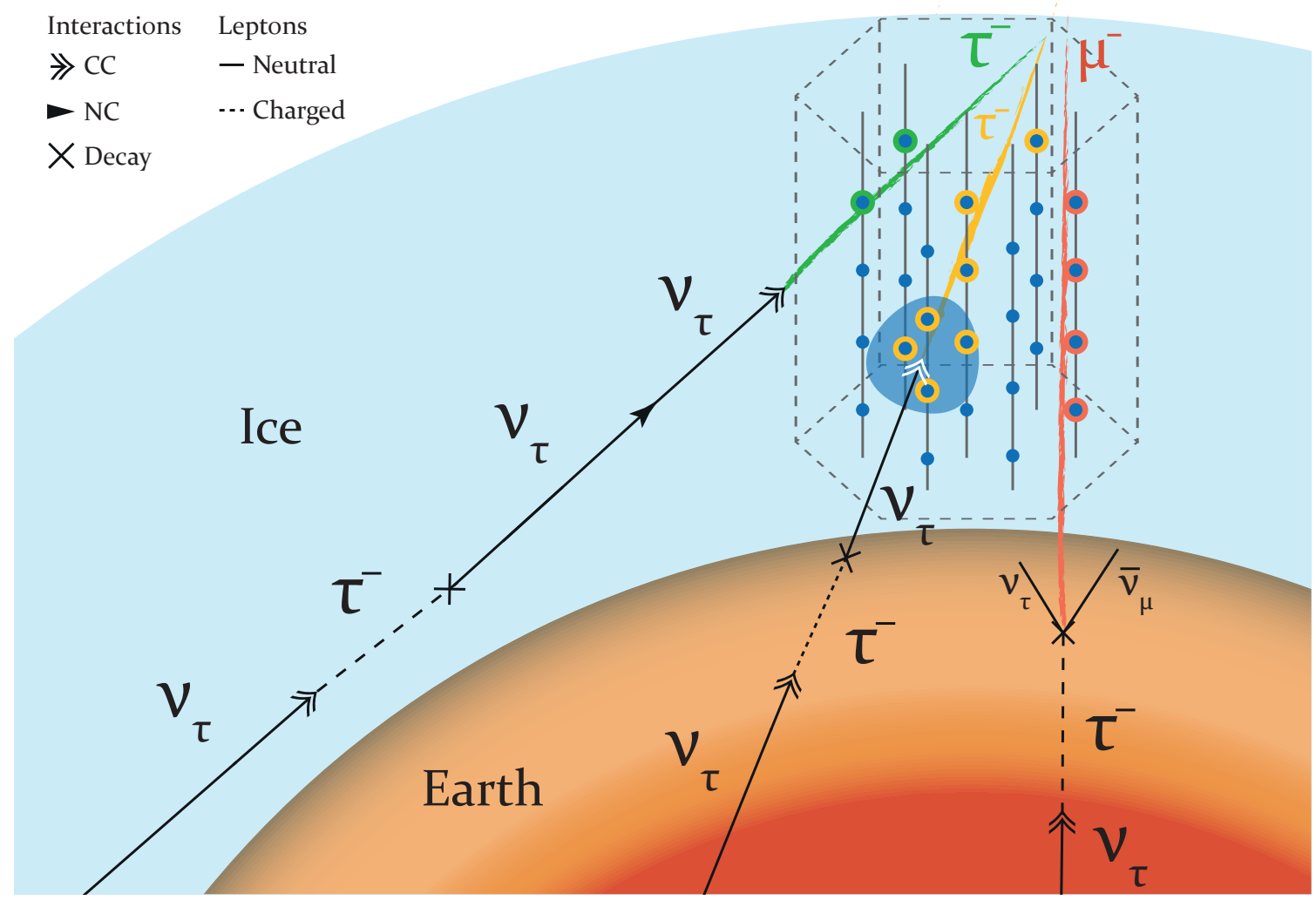

Figure 2: Schematic of lepton propagation through the Earth. There are three possible signatures from EeV tau-neutrino secondaries, described here from left to right. Left: A through-going tautrack, which is possible for taus at or above $10 \mathrm{PeV}$. Center: The interaction vertex is contained in the fiducial volume of the detector in this case, producing a cascade from the charged-current interaction, along with an outgoing tau track. Right: The tau decays before reaching the detector, producing a muon in $\approx 18 \%$ of the cases, which can subsequently enter the detector.

accept or reject procedure, either Neutral- (NC) or Charged (CC)- current process is chosen at the point of interaction. The energy loss in each process is sampled from the corresponding differential cross sections. For NC, the neutrino continues, and a new free-streaming distance is sampled. For $\mathrm{CC}$, a tau is created which is then propagated using MMC. The final tau energy and distance traveled are recorded, at which point it decays. The energy of the daughter tau-neutrino is sampled from the tau decay distribution, adopting the parametrization in [19]. From there, the leading tau-neutrino is tracked and the process repeats. Other secondary neutrinos (eg. from tau/muon decays) have a much softer energy spectrum and are neglected here. The algorithm stops when the leading particle emerges from the medium, at which point the particle identity and final energy are recorded, and if requested by the user, a detailed history of losses and interactions.

\subsection{Emerging secondary flux}

Using TauRunner, we calculate the resulting tau and neutrino energy distributions after traversing the Earth. We test a range of incident neutrino angles to vary the column depth, and choose one energy value per energy decade from $100 \mathrm{GeV}$ to $1 \mathrm{ZeV}$. We find that secondary neu- 
trino spectra are identical for steep incident angles (above 20 degrees below the horizon) and energies above an $\mathrm{EeV}$. This primary energy degeneracy begins to manifest when neutrinos undergo 2 or more charged current interactions in the Earth, or in other words when the width of the first interaction point is much shorter than the traversed chord length. The main reason for this degeneracy is the tau losses. Because photo-nuclear interactions scale roughly with the energy of the tau, higher energy taus lose more energy per column density traveled. This causes the tau range to asymptote above a threshold energy determined by the energy loss model used. Then, the tau reaches a point where decay is again dominant, which sets a lower limit on the tau energy before decay. Therefore, as long as the tau is created at or above $1 \mathrm{EeV}$, it will decay around $10-100 \mathrm{PeV}$ and travel the same distance on average. This, in turn, causes emerging secondary neutrino spectra to be identical, and the small differences are due to the variation in the primary neutrino interaction length. Although this is an important subtlety for Earth-skimming neutrinos [16, 19, 20, 21, 22, 23], it becomes negligible for large enough distances.

We show secondary neutrino energy distributions after propagation through the earth in Figure 3. Here we fix the emergence angle to be 30 degrees below the horizon. The gray line is the expected transmission probability calculated using exponential suppression, where the exponent is the ratio of the propagated distance to the neutrino mean interaction length. Peaks in the bins represent the fraction of surviving neutrinos, and agree well with the expectation.

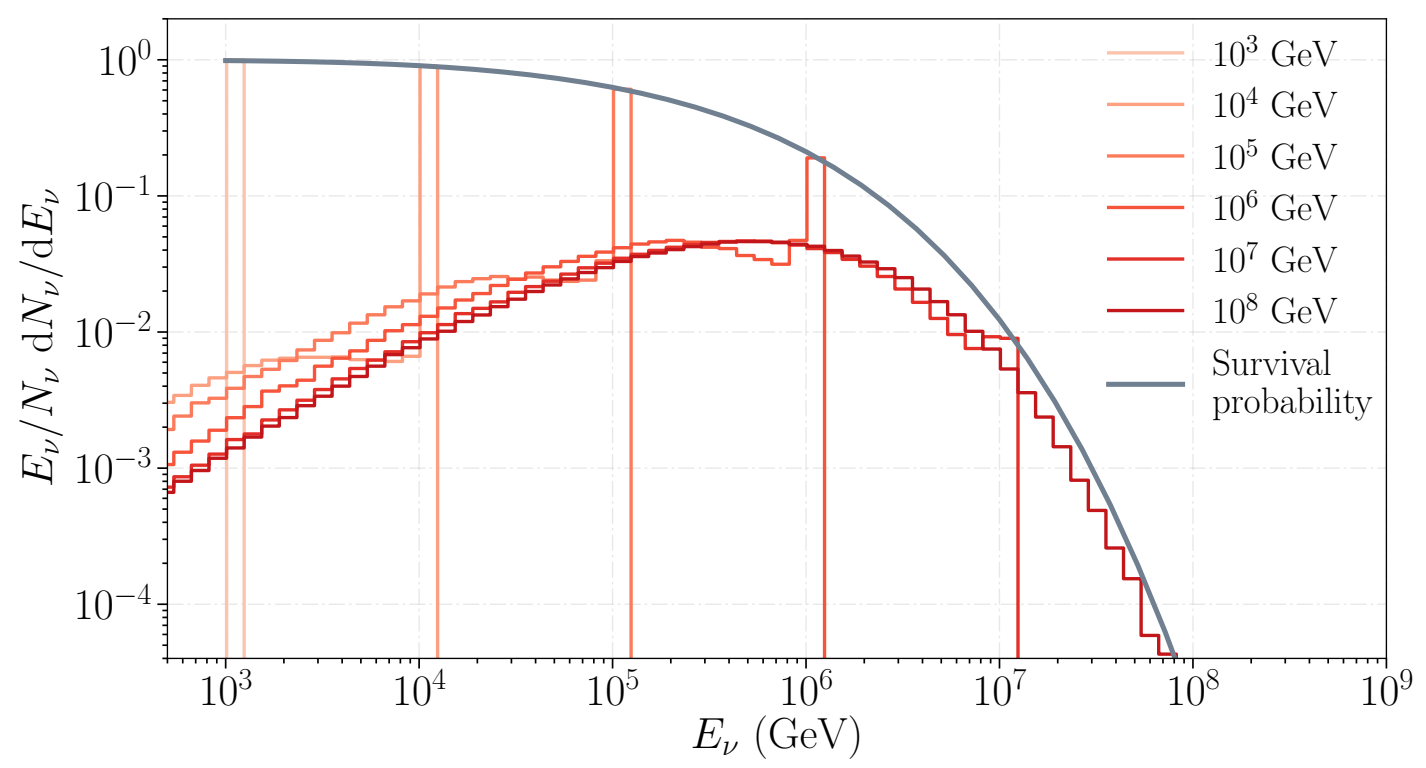

Figure 3: Mono-energetic tau neutrinos are injected at a set of initial energies (specified in the legend) and propagated through Earth to calculate the resulting spectrum as they emerge. The spike in each distribution represents the fraction of neutrinos that did not interact, while the secondary energy spectrum is represented by the curve to the left of each spike. The gray line shows the expected survival probability of the primary flux calculated for the same column depth. 


\section{ANITA and its anomalous events}

The ANtarctic Impulsive Transient Antenna (ANITA) collaboration has reported the detection of two events that at first glance are consistent with an upgoing astrophysical tau-neutrino [24]. For this to be the case, an incident tau-neutrino needs to undergo a CC interaction wherein the produced tau decays in the atmosphere producing an extensive air shower (EAS). Downward moving cosmic rays can also induce EAS, but the radio signals from these EAS acquire a phase reversal upon reflection on the ice, whereas the detected events do not display this phase reversal. If taken to be caused by tau-neutrinos, these events are unlikely from an isotropic flux [25, 26, 27], but localized emission could evade these constraints. Numerous beyond Standard Model explanations have also been suggested, running the gamut from sterile neutrinos [27, 28] to axion-photon conversion [29] or heavy BSM particle decays [30, 31]. Here, we show that the hypothesis of localized emission can also be ruled out by the non-observation of $\mathrm{TeV}-\mathrm{PeV}$ neutrinos with IceCube.

For an incident neutrino flux $\Phi\left(E_{V}\right)$, the number of expected events detected by ANITA due to tau showers in the atmosphere is given by

$$
N_{v}=\int d E_{v} d E_{v}^{\prime} \Phi\left(E_{v}\right) \frac{d N_{v}}{d E_{v}^{\prime}}\left(E_{v}^{\prime} ; E_{v}\right) \xi_{a c c}\left(E_{v}^{\prime}\right) \Delta T,
$$

where $d N\left(E_{v}^{\prime}\right) / d E_{v}^{\prime}$ is the energy distribution of secondary tau-neutrinos near the ice surface, $\Delta T$ is the duration of observation, and $\xi_{a c c}\left(E_{v}^{\prime}\right)$ is the ANITA acceptance which encodes the probability of neutrinos interacting in the ice, as well as the probability of a tau decay shower being induced in the upper atmosphere where ANITA is most sensitive [25]. To remove the Earth absorption effects present in the reported differential acceptance, we set the acceptance at all angles to be that at the horizon. For the incoming flux, we begin with a delta function in energy, $\Phi\left(E_{v}\right)=\Phi_{0} \delta\left(E_{v}-E_{0}\right)$, where $\Phi_{0}$ is the normalization. We tune the primary energy, $E_{0}$, to result in the maximum tau exit probability which subsequently causes more events detected by ANITA. We find this energy to be $1 \mathrm{EeV}$

As was discussed earlier, because of the steep zenith angle of AAE141220, this incident flux of EeV neutrinos is guaranteed to manifest at Earth's surface predominately as a flux of TeV - PeV neutrinos. In order to find the maximum allowed normalization of the ANITA flux, we propagate both our delta function spectrum as well as the reported IceCube diffuse astrophysical flux from the High Energy Starting Event selection (HESE) [32] using TauRunner. Secondary spectra at the IceCube detector from both of these fluxes are then compared, finding the $90 \%$ C.L upper limit by ensuring that the ANITA flux does not exceed the measured diffuse flux at more than $90 \%$ C.L. Results are shown in figure 4 . We place limits on the time-integrated flux as the time profile of the intrinsic flux is unknown. Ultimately, we find that the flux required to produce one event at ANITA and that allowed by IceCube measurements are orders of magnitude apart, making it highly unlikely for the reported event to be a tau-neutrino whose origin is astrophysical.

\section{Summary}

In this work we investigate the anomalous ANITA events using a monte carlo package, TauRunner, that calculates neutrino and tau distributions after propagating through the Earth. We find that incoming neutrinos with energies above an $\mathrm{EeV}$ emerge from the Earth at energies between $100 \mathrm{TeV}$ 


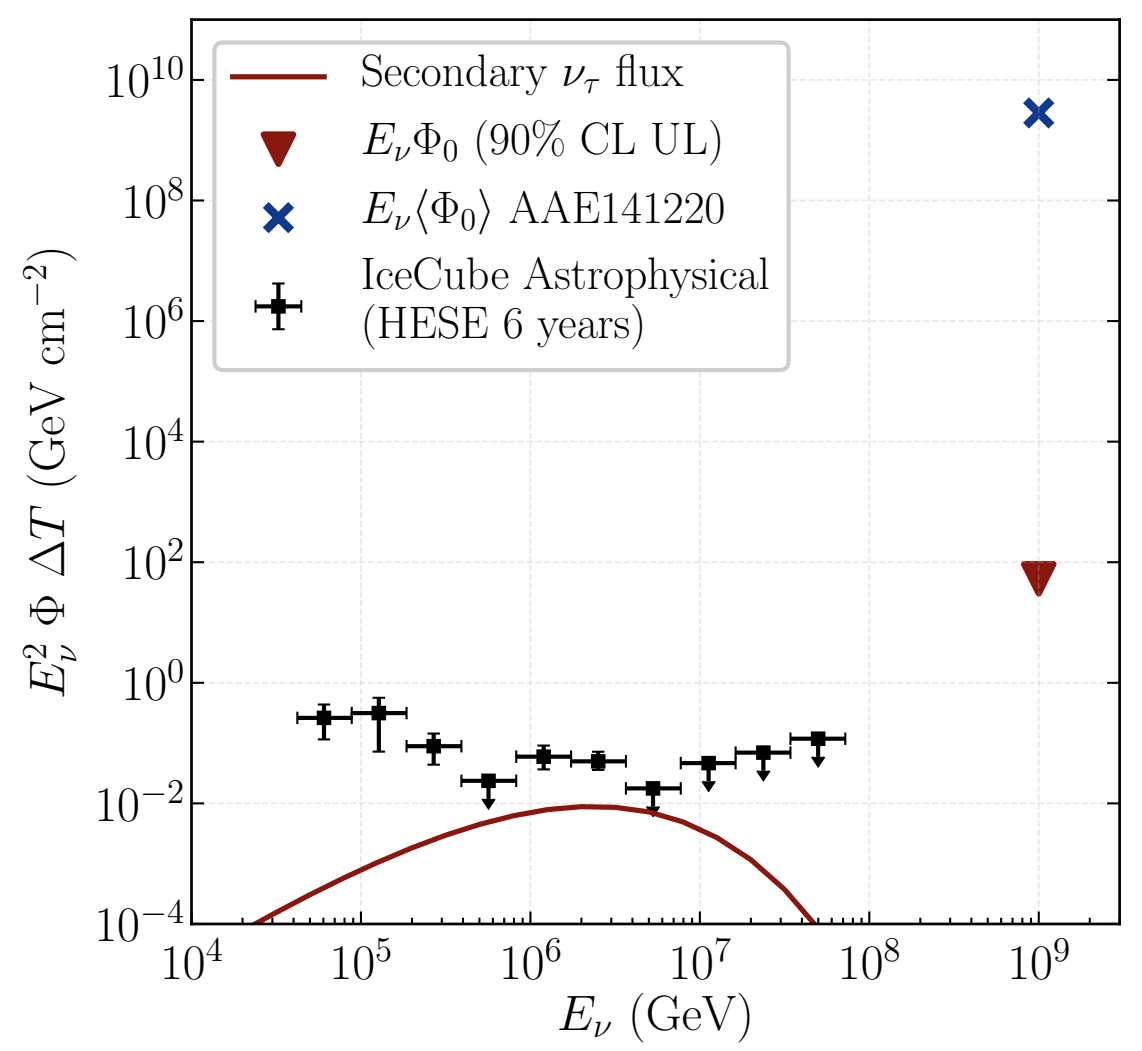

Figure 4: Maximum allowed flux of EeV neutrinos (maroon arrow), given an injected monoenergetic neutrino flux at or above the detected ANITA event energy (AAE141220). The normalization of the secondary flux is set to the maximum that does not exceed IceCube's diffuse astrophysical flux. The flux needed to produce one event in the third flight of ANITA (blue marker) exceeds the upper limit by many orders of magnitude.

and $10 \mathrm{PeV}$, and manifest as upgoing tracks in IceCube. We use the recently detected ANITA events to demonstrate this technique, and find that a tau-neutrino interpretation of these events is inconsistent with the TeV-PeV flux detected by IceCube. This technique can be used to verify or reject any future $\mathrm{EeV}$ measurement at steep angles, and serves as a complement to detectors that are sensitive to energy ranges higher than IceCube's.

\section{Acknowledgements}

The authors would like to thank Kareem R.H.A.M Farrag for creating the schematic shown in Figure 2, and Dmitry Chirkin and Sam Fahey for useful discussions. 


\section{References}

[1] IceCube Collaboration, M. G. Aartsen et al., Phys. Rev. Lett. 113 (2014) 101101.

[2] ANITA Collaboration, P. W. Gorham et al., Phys. Rev. D98 (2018) 022001.

[3] ARA Collaboration, P. Allison et al., Astropart. Phys. 70 (2015) 62-80.

[4] K. Fang et al., PoS ( ICRC2 017 ) 996 (2018). [35,996(2017)].

[5] A. V. Olinto et al., PoS ( ICRC2017) 542 (2018). [35,542(2017)].

[6] F. Halzen and D. Saltzberg, Phys. Rev. Lett. 81 (1998) 4305-4308.

[7] MiniBooNE Collaboration, A. A. Aguilar-Arevalo et al., Phys. Rev. D81 (2010) 092005.

[8] NuTeV Collaboration, M. Tzanov et al., Phys. Rev. D74 (2006) 012008.

[9] P. Vogel and J. F. Beacom, Phys. Rev. D60 (1999) 053003.

[10] A. Kurylov, M. J. Ramsey-Musolf, and P. Vogel, Phys. Rev. C67 (2003) 035502.

[11] Super-Kamiokande Collaboration, Z. Li et al., Phys. Rev. D98 (2018) 052006.

[12] IceCube Collaboration, M. G. Aartsen et al., Nature 551 (2017) 596-600.

[13] C. A. Argüelles, et al., Phys. Rev. D92 (2015) 074040.

[14] M. M. Block, L. Durand, and P. Ha, Phys. Rev. D89 (2014) 094027.

[15] A. Cooper-Sarkar, P. Mertsch, and S. Sarkar, JHEP 08 (2011) 042.

[16] Y. S. Jeong, et al., Phys. Rev. D96 (2017) 043003.

[17] D. Chirkin and W. Rhode, hep-ph/0407075.

[18] A. M. Dziewonski and D. L. Anderson, Phys. Earth Planet. Interiors 25 (1981) 297-356.

[19] S. I. Dutta, M. H. Reno, and I. Sarcevic, Phys. Rev. D66 (2002) 077302.

[20] T. M. Venters, et al., arXiv:1906.07209.

[21] M. H. Reno, J. F. Krizmanic, and T. M. Venters, arXiv:1902.11287.

[22] S. I. Dutta, M. H. Reno, and I. Sarcevic, Phys. Rev. D62 (2000) 123001.

[23] S. I. Dutta, Y. Huang, and M. H. Reno, Phys. Rev. D72 (2005) 013005.

[24] ANITA Collaboration, P. W. Gorham et al., Phys. Rev. Lett. 121 (2018) 161102.

[25] A. Romero-Wolf et al., arXiv:1811.07261.

[26] D. B. Fox, et al., Submitted to: Phys. Rev. D (2018).

[27] S. Chipman, et al., arXiv:1906.11736.

[28] G.-y. Huang, Phys. Rev. D98 (2018) 043019.

[29] I. Esteban, et al., arXiv:1905.10372.

[30] A. Connolly, P. Allison, and O. Banerjee, arXiv:1807.08892.

[31] J. H. Collins, P. S. Bhupal Dev, and Y. Sui, Phys. Rev. D99 (2019) 043009.

[32] IceCube Collaboration, C. Kopper, PoS ( ICRC2017) 981 (2018). 